\title{
ELABORATION DU RÉFÉRENTIEL HYDROGRAPHIQUE D'HAÏTI A PARTIR D'UN MNT ASTER
}

\author{
Laurent Polidori ${ }^{1}$, Maxime Claden ${ }^{2}$, Romain Frelat $^{2}$, Mahmad Elhage $^{1}$, Fouzia Bendraoua ${ }^{2}$, Gary Doliscar $^{3}$, \\ Philémon Mondesir ${ }^{4}$, Boby Emmanuel Piard ${ }^{4}$, Cédric Gaucherel ${ }^{2}$ \\ 1: Laboratoire de Géodésie et Géomatique (CNAM / L2G), Le Mans, France \\ 2 : Institut Français de Pondichéry (IFP), Pondichéry, Inde
}

3 : Comité Interministériel d'aménagement du Territoire(CIAT), Port-au-Prince, Haïti

4 : Centre National de l'Information Géo-Spatiale (CNIGS), Port-au-Prince, Haïti

\begin{abstract}
Résumé
L'étude présentée dans cet article a conduit au premier référentiel hydrographique homogène sur Haïti. Une méthode automatisée a été mise en œuvre pour l'extraction du réseau hydrographique et des limites de bassins versants à partir d'un MNT ASTER et d'une carte topographique numérisée. Une analyse statistique des résultats obtenus a révélé les propriétés fractales du relief de la région et a aidé à fixer des seuils pour retenir les bassins versants les plus significatifs. Le contrôle de qualité du réseau hydrographique, réalisé à partir de points de contrôle et d'une comparaison visuelle avec les images de Google Earth, confirme une exactitude géométrique inférieure ou égale au pas d'échantillonnage du MNT initial (de l'ordre de $30 \mathrm{~m}$ ). Les bassins versants extraits ont en outre fait l'objet d'une codification adaptée, qui permet de fournir au gouvernement haïtien un référentiel hydrographique précis et homogène pour ses études ultérieures.
\end{abstract}

Mots clés : MNT ASTER, réseau hydrographique, Haïti.

\begin{abstract}
The study presented in this article has provided the first homogeneous hydrographical reference system on Haitti. An automated method was implemented for the extraction of the river network and the watershed boundaries based on an ASTER DTM and a digitized topographic map. A statistical analysis of the results highlighted the fractal properties of the relief of the region and thresholds were fixed to select the most significant watersheds. The quality control of the river network, based on ground control points and on a visual comparison with Google Earth imagery, highlighted a geometrical accuracy close to or better than the DTM mesh size (around $30 \mathrm{~m}$ ). In addition, the selected watersheds were classified with an adapted codification, which provided the Haitian government a precise and homogeneous hydrographical reference system for further studies.
\end{abstract}

Keywords : ASTER DTM, hydrographic network, Haitti.

\section{Introduction}

La gestion efficace des ressources en eau d'une région ou d'un pays exige une bonne connaissance du territoire et notamment de l'organisation spatiale de ses bassins versants. Une étude baptisée BVH (pour Bassins Versants d'Haïti) a été réalisée à la demande du gouvernement haïtien dans le but de fournir un référentiel hydrologique et hydrographique détaillé pour la gestion des ressources en eau de l'ensemble du pays (Frelat et al., 2012). Le CIAT (Comité Interministériel de l'Aménagement du Territoire), commanditaire de l'étude, ne disposait pas d'une cartographie suffisamment précise pour de nombreuses études du réseau hydrographique et des bassins versants. Dans ce but, une méthode a été mise en œuvre à partir d'un MNT (Modèle Numérique de Terrain) issu d'images ASTER et d'un réseau hydrographique vectoriel issu de cartes topographiques. Cette opération s'est appuyée sur des algorithmes classiques d'extraction de réseaux hydrographiques et de délimitation des bassins versants, qui ne présenteraient pas d'originalité particulière si le contexte n'avait exigé quelques adaptations.

D'une part, les recherches qui visent à tester ou optimiser des algorithmes de traitement de données géographiques sont souvent mises en œuvre sur des petites zones, dans le cadre de projets pilotes, avec des données suffisantes et des possibilités d'intervention par des personnes très qualifiées, donc pas forcément reproductibles. Cette situation classique permet d'améliorer les résultats localement et d'apprécier ce qu'une méthode de traitement permet d'offrir de mieux dans des conditions favorables, mais ne permet généralement pas une extrapolation sur de grandes étendues comme celle d'une région ou d'un pays. Au contraire, le besoin de produire un résultat sur l'ensemble du territoire haïtien nous 
a contraint à adopter une méthode en grande partie automatisable, et à nous satisfaire des rares données couvrant tout le territoire fournies par le CNIGS (Centre National de l'Information Géo-Spatiale).

D'autre part, le relief particulier du territoire haïtien, où des secteurs montagneux escarpés côtoient de vastes zones de plaine, met en échec les algorithmes classiques et a nécessité des ajustements de la méthode habituellement mise en œuvre, notamment par un creusement du réseau en région de plaine et une correction des cours d'eau aberrants que les logiciels disponibles ne permettaient pas de détecter de manière satisfaisante. Enfin, le résultat devant être livré aux services haïtiens de gestion de l'eau, nous avons adopté des critères de délimitation des bassins conformes aux standards de ces services, notamment en matière de superficie des bassins, les plus petits n'étant pas requis. Nous avons retenu les plus grands bassins versants à partir d'une analyse statistique de leurs superficies et d'un critère de seuil basé sur les propriétés fractales du relief.

De nombreuses études ont consisté à extraire le réseau hydrographique et les limites de bassins versants à partir d'un MNT (O'Callaghan et Mark, 1984; Tarboton et Rodriguez-Iturbe, 1991) et à caractériser localement le réseau au moyen d'indicateurs (Pella et al., 2012). Dans certains cas, la cartographie officielle est de qualité insuffisante ou hétérogène, et la possibilité de cartographier l'hydrographie du territoire pour un coût raisonnable dépend alors de la disponibilité d'un MNT gratuit. Ainsi l'élaboration du référentiel hydrographique sur la Guyane française s'est appuyée sur le MNT SRTM (Labbé et al., 2012). Quant à notre étude sur Haïti, elle s'est appuyée sur le MNT ASTER comme l'ont fait avant nous d'autres auteurs (Hosseinzadeh, 2011).

Après une description de la zone d'étude et des données utilisées, cet article présente la méthode mise en œuvre en insistant sur les innovations apportées. Les résultats obtenus sont présentés et font l'objet d'une discussion.

\section{Zone d'étude et données}

\subsection{Le territoire haïtien}

Haïti fait partie, avec la République Dominicaine, d'une île autrefois appelée Hispaniola, au sein de l'arc des Grandes Antilles (Figure 1). Le territoire haïtien couvre l'extrémité occidentale de l'île sur une superficie de 27750 km $^{2}$ et se prolonge par deux presqu'îles qui s'étendent vers l'ouest, l'une vers Cuba et l'autre vers la Jamaïque (CEGET, 1985). Le territoire est montagneux, on y distingue cinq massifs principaux et le point culminant est le pic de la Selle $(2680 \mathrm{~m})$. Les plaines (moins de $200 \mathrm{~m}$ ) ne représentent qu'un quart de la surface totale du pays environ.

La tectonique active et la conjonction entre des précipitations importantes et une couverture végétale fortement dégradée font d'Haïti un pays particulièrement exposé aux risques d'inondations, tremblements de terre, érosions, glissements de terrain, notamment. La déforestation importante, qui résulte de la forte pression démographique depuis le début du XIXème siècle pour laisser la place à des cultures de rente, accentue encore l'érosion et constitue un fléau à l'effet dévastateur sur la ressource en eau (Anglade, 1974).

\subsection{Données utilisées}

Différentes données ont été testées, mais seules celles qui étaient disponibles sur l'ensemble du territoire et possédaient une précision suffisante ont été retenues :

- Un modèle numérique de terrain produit par traitement photogrammétrique d'images du capteur ASTER (Advanced Spaceborne Thermal Emission and Reflection Radiometer) avec un pas au sol d'un arc-seconde (environ $30 \mathrm{~m}$ à l'équateur). II s'agit du produit ASTER GDEM v2, à maille carrée, réalisé par corrélation automatique sur la majeure partie des terres émergées et accessible en ligne ${ }^{1}$.

- Une couche vectorielle contenant le tracé des rivières principales et secondaires d'Haïti, et issue de la numérisation d'une ancienne carte topographique au 1:50000. Celle-ci constituait jusqu'alors le réseau hydrographique de référence le plus complet qu'Haïti eût en sa possession, et une analyse sur quelques sites, comme nous alIons le voir plus loin, a montré qu'elle était fiable en planimétrie.

Nous nous sommes en outre appuyés sur des images Quickbird disponibles dans Google Earth et sur des levés par GPS pour apprécier la qualité des résultats obtenus en termes d'exactitude planimétrique.

\section{Méthode mise en œuvre}

\subsection{Extraction du réseau hydrographique}

Le réseau hydrographique du territoire haïtien a été extrait à partir du MNT ASTER et amélioré par l'usage du fichier hydrographique vectoriel. De nombreux algorithmes d'extraction du réseau hydrographique ont été développés et publiés. La méthode la plus usuelle est basée sur l'algorithme de O'Callaghan et Mark (1984) qui détermine la direction du flux dans une grille à maille carrée en fixant arbitrairement l'aire d'accumulation qui donne naissance à un cours d'eau. Les biais directionnels qui étaient souvent observés ont suscité des travaux visant à les éviter, par exemple en injectant une donnée vectorielle externe avec l'algorithme du «stream burning» (Saunders, 1999).

La plupart des méthodes font des hypothèses simplificatrices, que nous avons adoptées également, qui consistent en particulier à négliger les infiltrations

\footnotetext{
1. http ://eros.usgs.gov/\#/Find_Data/Products_and_Data_Available/gtopo30/hydro/namerica
} 


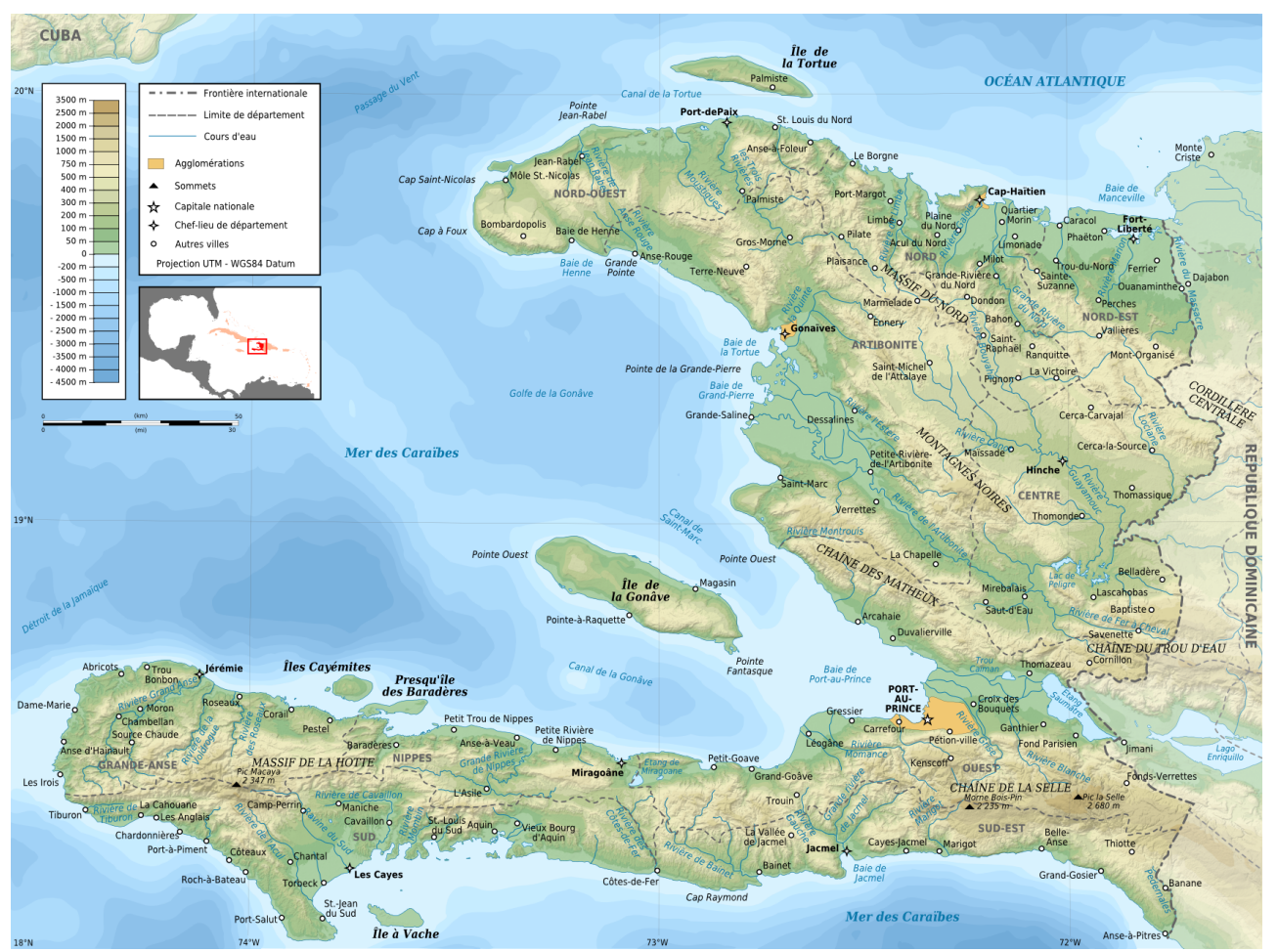

FIGURE 1 : Localisation géographique d'Haïti (Source : Wikipédia, 2013).

dans le calcul de l'accumulation de l'eau, et à assimiler le réseau hydrographique au réseau des talwegs (et de manière duale les limites de bassins versants aux lignes de crêtes).

La méthode mise en œuvre passe par plusieurs étapes successives (Figure 2). La première étape consiste à calculer les directions d'écoulements à partir du MNT. II est ensuite possible d'extraire le réseau par l'intermédiaire du calcul de l'accumulation des flux d'eau et d'un nettoyage détaillé plus loin. Enfin, les limites des bassins versants sont extraites à l'aide d'un algorithme utilisant les directions d'écoulement pour déceler les lignes de crête dans le MNT.

Nous avons utilisé pour le calcul des directions d'écoulement l'algorithme D8, disponible dans ArcGIS 9.3. Cet algorithme est le plus couramment employé pour les études de grande ampleur (sur l'ensemble d'une région ou d'un continent), en raison de sa simplicité et de sa robustesse (Charleux-Demargne, 2001). Cependant, s'il donne généralement des résultats satisfaisants en zone montagneuse, il peut générer des artéfacts liés à l'utilisation des huit pixels voisins du raster pour la simulation du ruissellement. Notamment en zone de plaine, l'amplitude altimétrique est souvent inférieure à l'incertitude du MNT ASTER, ce qui affecte la qualité de l'extraction du réseau hydrographique (Gyasi-Agyei et al., 1995; McMaster, 2002). Des méthodes existent pour améliorer la qualité des MNT dans les zones plates (Callow et al., 2007). Les solutions proposées dans la

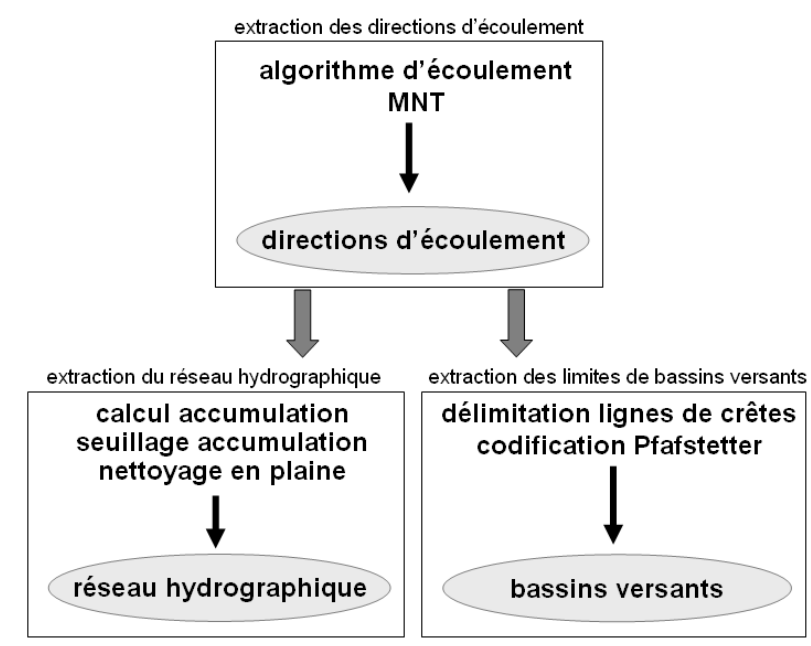

FIGURE 2 : Organigramme général du processus d'extraction du réseau hydrographique et des limites des bassins versants. 
littérature ont en commun d'utiliser une donnée exogène permettant de compléter le MNT lorsque celui-ci ne renseigne pas correctement sur l'hydrographie. Dans cet esprit, nous avons eu recours au fichier vectoriel des rivières principales, moins dense dans l'ensemble du pays notamment en région montagneuse, mais plus fiable en plaine.

Ainsi, nous avons combiné les avantages respectifs du MNT ASTER (en montagne surtout) et de la donnée vectorielle (en plaine surtout) par un algorithme de "stream burning» ou marquage des rivières, qui consiste à modifier le plan altimétrique du MNT en incrustant une donnée hydrographique externe. Le principe général est d'abaisser d'une valeur arbitraire l'altitude des points du MNT qui se trouvent sur le tracé d'un réseau hydrographique de référence. Nous avons utilisé ce principe pour adapter le MNT ASTER, en y incrustant le tracé vectoriel, sans réaliser de lissage à proximité du couloir d'abaissement. Les tronçons extraits ont ensuite fait l'objet d'une classification selon les ordres de Strahler.

Afin de ne retenir que les talwegs les plus significatifs sur le plan hydrologique, il est nécessaire de n'amorcer la classification des ordres qu'à partir d'un certain seuil sur l'aire du bassin élémentaire collectant l'eau de pluie. Nous avons choisi d'utiliser un seuil unique pour tout le territoire haïtien, pour conserver la cohérence du futur référentiel. Dans une phase exploratoire, nous avons calculé et seuillé les aires de drainage en amont de chaque pixel, testant des valeurs de seuil de 20, 10 et 5 pixels (correspondant respectivement à des aires de drainage de 18540,9270 et $4635 \mathrm{~m}^{2}$ ). Des observations en zone de relief à proximité des sources nous ont permis de retenir empiriquement le seuil de 10 pixels, compromis entre l'intégration de petits artéfacts (seuil de 5 pixels) et un début de réseau trop éloigné des sources (seuil de 20 pixels). L'utilisation d'un seuil unique optimisé pour les régions montagneuses engendre en plaine de nombreux tronçons irréalistes. Le réseau extrait automatiquement a donc fait l'objet d'un nettoyage, basé sur l'hypothèse que très peu de rivières prennent leur source en plaine. C'est une hypothèse forte, mais qui est vérifiée dans la très grande majorité des cas, en particulier pour Haïti au relief presque bimodal (avec un mode en plaine et un autre en montagne). Elle conduit à supprimer les tronçons caractérisés par une faible pente et par un ordre de Strahler égal à 1 (correspondant à une source).

La méthode de nettoyage est un filtre itératif sur ces deux critères (Figure 3). À partir du réseau extrait, l'ordre et la pente sont calculés pour chaque tronçon. Ensuite, tous les tronçons d'ordre 1 avec une pente inférieure ou égale à un certain Seuil de Pente (SP) sont supprimés. Un nouveau réseau est alors obtenu. S'il est identique au réseau initial (c'est-à-dire que l'on ne peut plus améliorer le nettoyage), ce réseau est le résultat final de notre nettoyage. Sinon, une nouvelle itération est appliquée. Le nombre d'itérations ne peut excéder l'ordre maximal de notre réseau initial. Notons qu'à chaque itération, les ordres des tronçons sont susceptibles de changer, mais pas la pente. Cette méthode de nettoyage a été appliquée sur le réseau initial, avec un seuil de pente variant de $S P=0,02$ à 0,12 par pas de 0,01 . Un simple examen visuel sur des images de Google Earth a permis d'apprécier l'influence du seuil de pente sur la moitié nord de l'île (zone test supposée représentative d'Haïti). Une valeur SP $=0,05$, permettant d'éliminer correctement les petits cours d'eau irréalistes en plaine et de conserver un réseau dense en montagne, a été établie empiriquement et appliquée, par des calculs rapides, à tout le réseau d'Haïti.

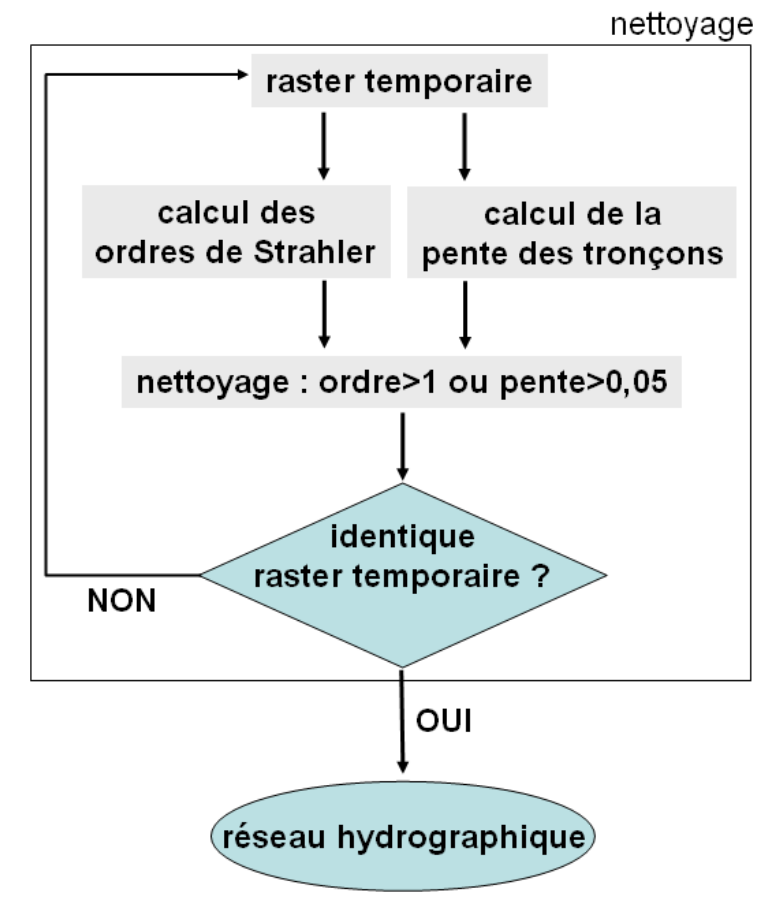

FIGURE 3 : Étapes successives de la méthode de nettoyage du réseau extrait, avec un seuil de pente égal à $S P=0,05$.

Ce processus itératif permet de supprimer les tronçons d'ordre supérieur à 1 qui n'ont pas de réalité physique, car prenant leur source en plaine (Figure 4). Ce nettoyage ne crée pas de discontinuités entre la plaine et les zones montagneuses. Ce processus innovant, qui n'a pas été retrouvé dans la littérature, s'est avéré satisfaisant pour notre étude.

\subsection{Délimitation des bassins versants}

La délimitation des bassins versants a utilisé l'outil «Basin» du logiciel ArcGIS 9.3, qui s'appuie sur l'image raster des directions d'écoulement. II reconnaît l'exutoire de chaque bassin quand la direction d'écoulement rencontre le trait de côte. À partir de ces points, l'algorithme 


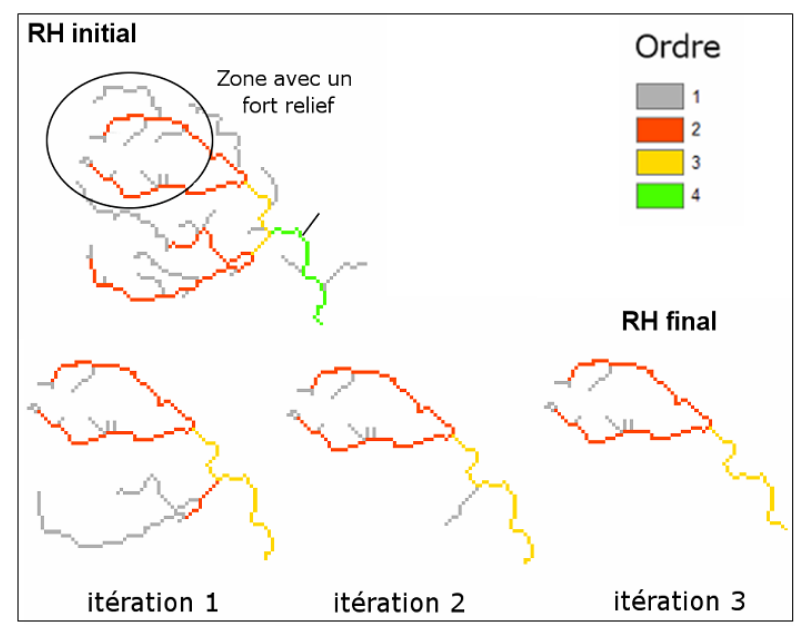

FIGURE 4 : Filtre itératif sur la pente et l'ordre de Strahler.

parcourt les lignes de crête et définit ainsi les limites des bassins.

Cet outil est simple et efficace. Cependant, il crée de très nombreux bassins versants côtiers qu'il convient d'éliminer, soit parce qu'ils ne sont pas réalistes, soient parce qu'ils ne présentent pas d'intérêt pour la gestion de la ressource en eau. Ne disposant pas de données sur la position réelle des exutoires, nous avons procédé par seuillage sur l'aire des bassins afin de ne conserver que ceux qui ont une aire significative. Ayant classé les bassins par ordre décroissant d'aire, nous avons déterminé l'aire cumulée en fonction de leur ordre. Une représentation bi-logarithmique de cette relation présente souvent une tendance linéaire représentative d'un comportement fractal du relief d'un bassin versant, même si elle est ici tracée pour plusieurs bassins d'une même région (Rodriguez-Iturbe et Rinaldo, 1997). Cette propriété a permis de mettre en évidence un changement de pente qui permettrait de séparer les grands bassins, correctement restitués et possédant une réalité hydrographique, et les plus petits bassins présentant peu d'intérêt et probablement fortement affectés par les imprécisions du MNT et par le comportement de l'algorithme de délimitation.

\subsection{Evaluation de la qualité géométrique du réseau hydrographique extrait}

La plupart des MNT disponibles sur de grandes étendues sont obtenus à partir d'imagerie spatiale, le plus souvent par photogrammétrie, et dans une moindre mesure par radargrammétrie et interférométrie radar. Or, toutes ces techniques tendent à optimiser la localisation absolue des points du MNT, appréciée généralement par l'erreur quadratique moyenne des altitudes déduite de points de contrôle, mais ce critère de qualité ne garantit nullement la précision des dérivées de l'altitude (pente, orientation, courbure) ni la fidélité aux formes de relief
(Polidori, 1995 ; El Hage, 2012).

Les imperfections du réseau hydrographique extrait d'un MNT dépendent des méthodes mises en œuvre pour la production du MNT (génératrices de bruit, lissage, artefacts) et pour l'extraction du réseau. Elles peuvent être de natures multiples, que l'on peut regrouper en erreurs géométriques et erreurs topologiques. Si la cohérence topologique est assurée par le principe même de l'algorithme d'extraction, la qualité géométrique du réseau dépend de la qualité des données utilisées et ne peut être évaluée sans une référence. En l'absence d'un réseau de référence, nous avons eu recours à des images de Google Earth combinées à des relevés de terrain par GPS. Nous avons créé pour cette évaluation un semis de 100 points disposés aléatoirement sur le réseau extrait dans le secteur nord d'Haïti, où l'on trouve des zones de plaine et de montagne, donc supposé représentatif de la morphologie d'ensemble du réseau hydrographique haïtien. L'intérêt de l'échantillonnage aléatoire des points est notamment de sélectionner aléatoirement les ordres des tronçons pour éviter d'y introduire des biais.

L'évaluation consiste tout d'abord à estimer l'erreur géométrique globale en mesurant pour chaque point la distance entre le réseau extrait et le réseau observable dans les images. Les images ont une résolution suffisante pour permettre de reconnaître la plupart des cours d'eau, toutefois cette reconnaissance a exigé pour chaque point un avis d'expert.

\section{Résultats et discussion}

\subsection{Réseau hydrographique et limites de bassins versants extraits}

La méthode mise en œuvre a permis de déterminer le réseau hydrographique et les limites de bassins versants de l'ensemble du territoire haïtien avec un niveau de détail bien supérieur à ce qui était disponible auparavant.

Ces résultats ont montré que les méthodes d'extraction et de correction étaient efficaces en zone de plaine, puisqu'il a été possible d'éliminer de façon automatique les petits cours d'eau non réalistes (prenant leurs sources en zone de plaine) tout en conservant les rivières principales. Le seuil de pente $\mathrm{SP}$ a été fixé par tâtonnement. Une illustration en rive droite d'une confluence de la Quinte où le relief est très faible, montre qu'une valeur trop élevée $(S P=0,1)$ aurait conduit à supprimer également des cours d'eau ayant une réalité physique (Figure 5).

\subsection{Caractéristiques statistiques du réseau hydro- graphique}

Le réseau extrait présente des caractéristiques classiques liées aux propriétés fractales du relief terrestre, 
et respecte notamment la loi de Horton selon laquelle le nombre total des tronçons d'ordres successifs (ici, de l'ensemble du pays) décroît selon une progression géométrique simple. Le logarithme de ce nombre présente donc une décroissance linéaire (Figure 6).

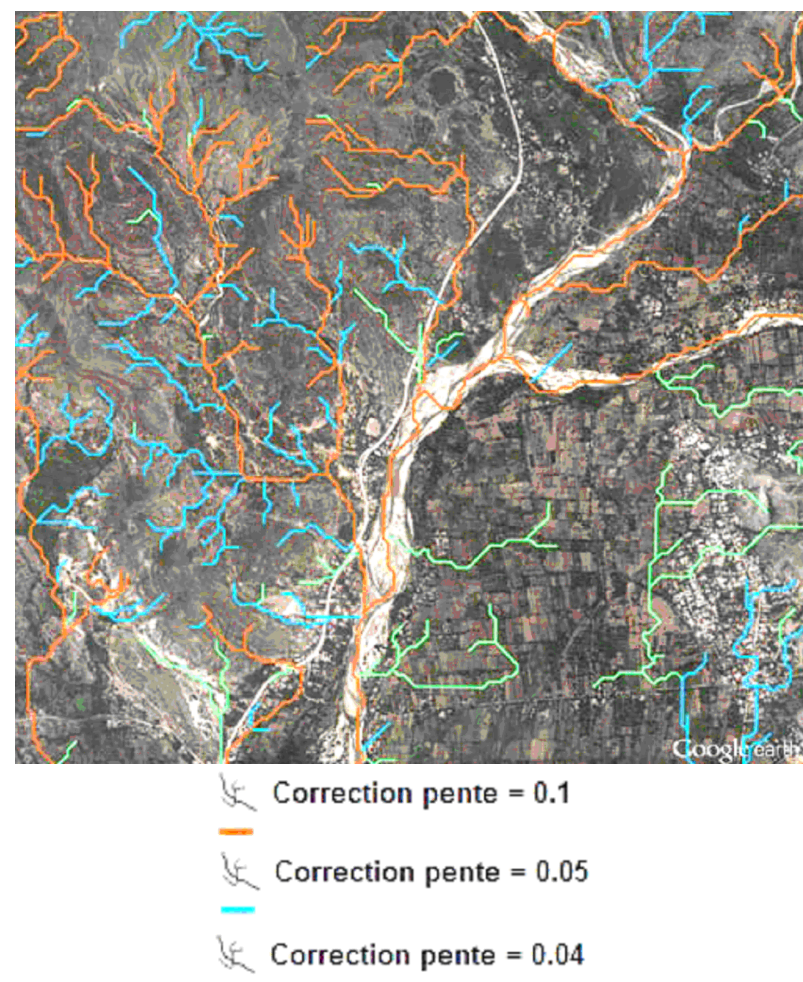

FIGURE 5 : Superposition des réseaux corrigés avec différentes valeurs de SP ( 0,1 en orangé ; 0,05 en bleu; 0,04 en vert) sur une zone de confluence de la rivière la Quinte. Le fichier vectoriel initial ne montrait qu'un cours d'eau (en blanc).

La droite de régression, calculée sans l'ordre 10 qui ne correspond qu'à un seul tronçon, présente une pente de $-0,79$ liée à la dimension fractale du réseau et donc à sa densité en Haïti $\left(R^{2}=0,9784\right)$. Les écarts à cette loi (non linéarité) révèlent souvent des phénomènes géomorphologiques particuliers, à l'exception des échelles extrêmes. En effet, les tronçons d'ordre très élevé ou de très grande dimension spatiale correspondent généralement à des cours d'eau en nombre réduit, voire un seul (ici, l'ordre de 10), ce qui invalide la représentativité statistique des résultats. A l'autre extrême, les tronçons d'ordre 1 ou de très petite dimension spatiale sont souvent affectés par les imperfections du MNT initial (bruit ou lissage excessif) ou par les opérations d'interpolation induites par l'algorithme d'extraction (Polidori et al., 1991). Ce phénomène n'apparaît quasiment pas sur le réseau extrait, sans doute en raison du seuillage appliqué à l'aire du bassin versant élémentaire. Ce constat justifie les choix adoptés pour les paramètres de l'algorithme d'extraction.

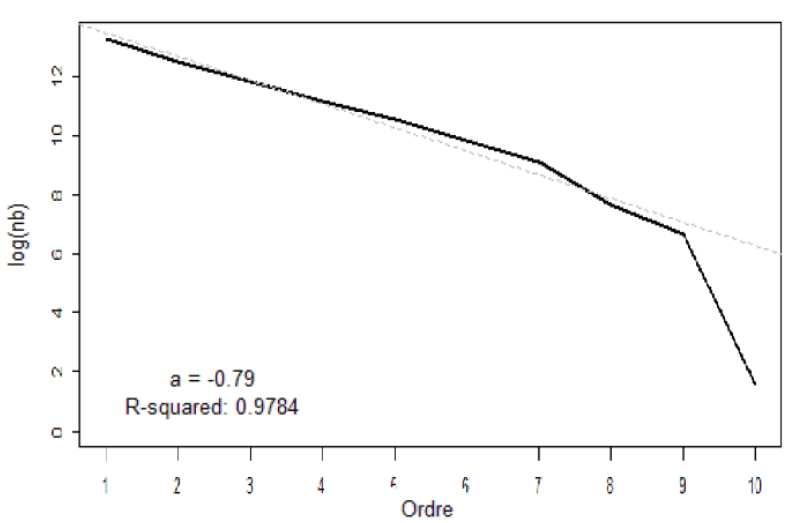

FIGURE 6 : Histogramme (représenté en échelle logarithmique) des ordres des tronçons de l'ensemble du réseau hydrographique d'Haïti.

\subsection{Caractéristiques statistiques des bassins ver- sants}

Les bassins versants extraits ont, de manière presque systématique, une aire inférieure à celle obtenue dans des études antérieures (Frelat, 2012). Ceci peut s'expliquer par le fait que nous délimitons les bassins versants au sens strict, avec un seul exutoire par bassin. La différence de surface correspond à des bassins de très petite taille, souvent sans réalité hydrographique et devant donc être éliminés. Pour déterminer le seuil au-dessous duquel les bassins versants doivent être éliminés, nous avons calculé l'aire cumulée par bassins versants de taille décroissante qui montre un comportement linéaire en logarithme pour les 75 plus grands bassins d'Haïti (Figure 7). Le changement de pente correspondant à une aire limite de $50 \mathrm{~km}^{2}$ $\left(R^{2}=0,9987\right)$. On retrouve ici, de façon flagrante dès le troisième bassin versant en taille, le comportement fractal du relief haïtien.

On constate également sur la figure 7 qu'après avoir pris en compte les 1275 plus grands bassins versants, le logarithme de la surface cumulée cesse de croître de manière significative. Cela signifie que l'aire cumulée des bassins suivants est négligeable. II s'agit souvent de bassins limités à un ou quelques pixels du MNT, dépourvus de signification hydrographique.

\subsection{Qualité géométrique du réseau hydrographique extrait}

En pratique, la mesure de distance entre le réseau extrait et celui observé n'est effectuée que lorsque la rivière observable a été correctement identifiée. Cela représente 67 points sur 100 dans cette étude. Dans les autres cas, il n'a pas été possible d'identifier correctement la rivière (végétation dense, contraste insuffisant, couverture nuageuse, rivière trop étroite compte tenu de la résolution...), ou encore l'extraction du réseau est manifestement erronée, notamment en zone 


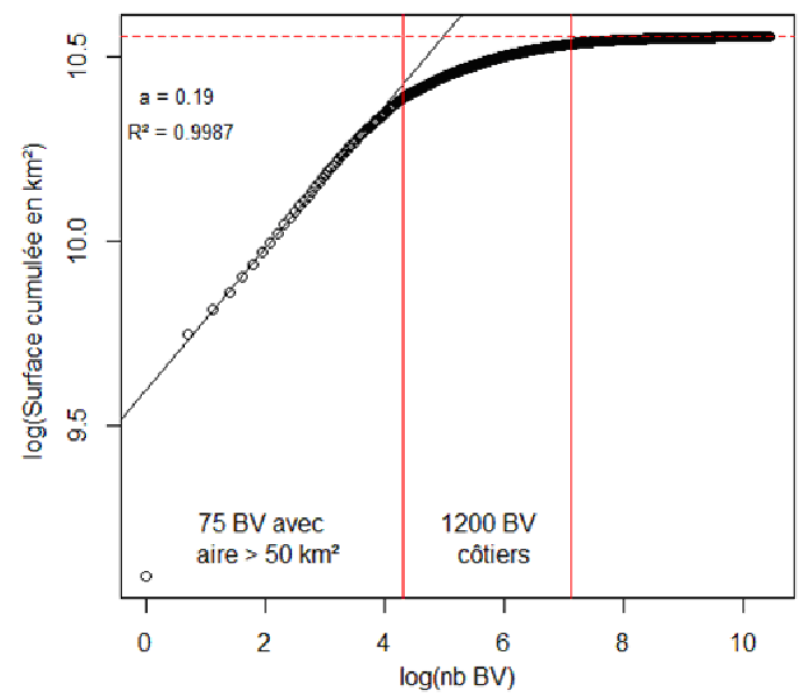

FIGURE 7 : Surface cumulée (en échelle logarithmique) des bassins versants haïtiens extraits de taille décroissante (à l'abscisse $\mathrm{N}$ est associé le logarithme de l'aire totale des $\mathrm{N}$ plus grands bassins versants).

anthropisée. Sur les 67 points mesurés, la distance moyenne est de $28 \mathrm{~m}$, et $95 \%$ des points mesurés sont à moins de $80 \mathrm{~m}$ de la rivière observable (Figure 8 ).

Ces résultats doivent être interprétés avec prudence, car l'exactitude des données de Google Earth n'est pas garantie, ne faisant l'objet d'aucun cahier des charges. Cependant, leur usage très répandu a conduit de nombreux utilisateurs à s'interroger sur leur qualité (Potere, 2008 ; Yousefzadeh er Mojaradi, 2012). Les études publiées s'accordent à constater une exactitude meilleure que $50 \mathrm{~m}$. L'exactitude, qui caractérise l'écart entre des données contrôlées et la réalité, est évaluée en termes d'erreur moyenne quadratique. Toutefois la qualité des données n'est pas homogène, et il semble que la précision de localisation soit meilleure dans les pays développés que dans les pays en développement, et qu'elle se soit globalement améliorée depuis 2008 avec la mise en ligne systématique des images de Quick Bird et World View, dont les erreurs de localisation sont de l'ordre de $20 \mathrm{~m}$ et $5 \mathrm{~m}$, respectivement, sans points d'appui. Cette expérience accumulée par les utilisateurs et les études publiées sur le sujet suggèrent que le réseau extrait en Haïti est entaché d'une erreur de localisation n'excédant pas quelques dizaines de mètres.

Compte tenu de ces incertitudes, l'évaluation a été complétée par une mission de terrain effectuée par les services haïtiens du CIAT et du CNIGS. Nous avons identifié 61 points se trouvant sur des ponts, et permettent donc de préciser par GPS la position des rivières. La distance moyenne entre le réseau extrait et la référence est de $22 \mathrm{~m}$ et $95 \%$ des écarts observés sont inférieurs à $47 \mathrm{~m}$. La distance maximale mesurée est de 74 m (Figure 8). Les contrôles basés sur des images de Google Earth et sur des relevés GPS ont donc conduit à des résul- tats comparables. Les erreurs de positionnement sont du même ordre de grandeur que (voire inférieures à) la maille du MNT ASTER utilisé pour l'extraction (30 m).

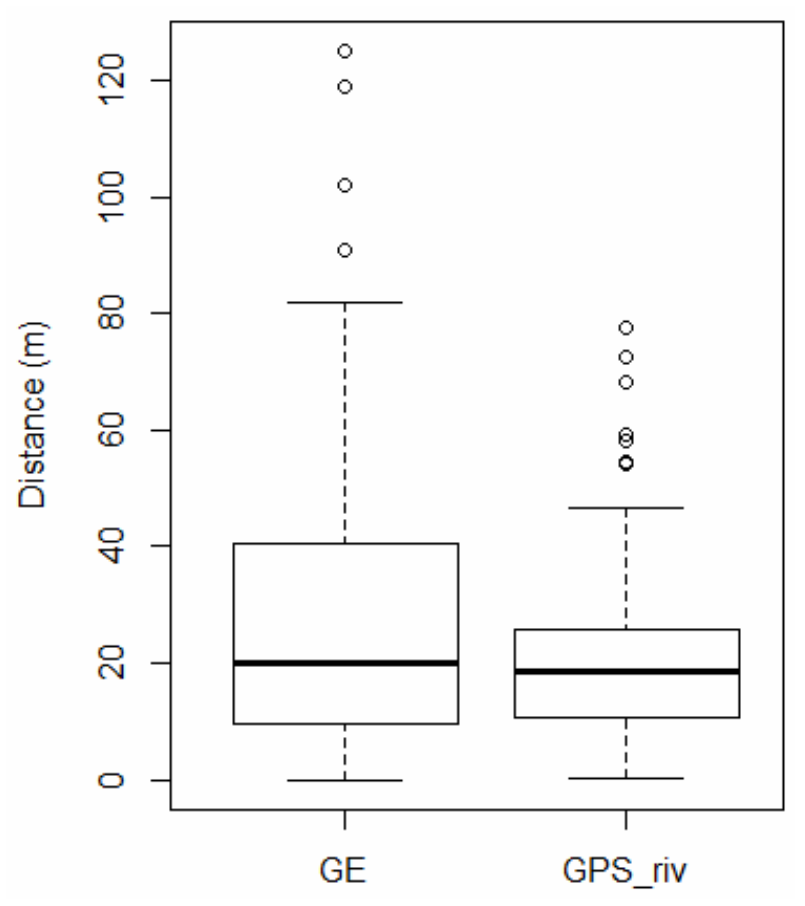

FIgURE 8 : Distribution des distances entre des points sélectionnés aléatoirement sur le réseau extrait dans le nord d'Haïti, et les positions de référence déterminées dans les images de Google Earth (67 observations, écart moyen de $28 \mathrm{~m}$ ) et levées par GPS (61 observations, écart moyen de $22 \mathrm{~m}$ ).

\section{Codification des bassins versants}

Les données hydrographiques extraites, une fois validées en termes de cohérence topologique et d'exactitude géométrique, ont fait l'objet d'une codification destinée aux gestionnaires haïtiens. Nous avons adopté la codification de Pfafstetter, couramment utilisée pour les projets de modélisation hydrologique (Verdin et Verdin, 1999), notamment à l'échelle continentale (De Jager et Vogt, 2010). Cette codification qui s'appuie sur le système décimal est arborescente : les plus grands bassins versants sont codés avec un chiffre, mais il est possible de les subdiviser en sous-bassins versants en ajoutant un chiffre au code initial. Tous les bassins de plus de $50 \mathrm{~km}^{2}$, les 75 plus grands, ont été codés en suivant cette méthode. Leur codage nécessite entre un chiffre, pour les plus grands, et cinq chiffres, pour les plus petits. L'origine de la codification a été choisie au point de rencontre entre la frontière Haïti-République Dominicaine et la Mer des Caraïbes (Figure 9). 


\section{Conclusion}

Une méthode entièrement automatisée a été mise au point pour l'extraction du réseau hydrographique et des limites de bassins versants à partir d'un modèle numérique de terrain ASTER et d'une carte topographique numérisée. Cette méthode s'appuie sur des algorithmes classiques disponibles dans des logiciels SIG du marché, notamment pour le creusement du réseau en zone de plaine, et sur un algorithme de nettoyage mis au point par les auteurs pour la correction des cours d'eau aberrants. Cette méthode a été appliquée à l'ensemble du territoire haïtien, fournissant aux autorités du pays un référentiel hydrographique homogène. Une analyse statistique des résultats a permis de mettre en évidence un comportement fractal du relief et de fournir un critère d'exclusion des petits bassins versants, sans intérêt pour la gestion. La qualité géométrique du réseau extrait a été validée au moyen d'images de Google Earth et de levés GPS, mettant en évidence une exactitude comparable à (voire meilleure que) la maille du MNT ASTER (30 m). Enfin, la carte des bassins versants a fait l'objet d'une codification permettant une gestion ultérieure méthodique et efficace de la ressource en eau. Cette étude se poursuit dans le cadre du projet BVH (Bassins Versants d'Haïti) en s'appuyant sur le référentiel ainsi obtenu pour une caractérisation géomorphologique détaillée des bassins versants, en cours de publication. Cette caractérisation spatiale sera complétée par une caractérisation temporelle, hydrologique et climatologique, de la ressource en eau.

\section{Références}

Anglade G., 1974. L'espace haïtien. Presses de l'Université du Québec, Montréal, Canada.

Callow J.N., Niel K.P.V., 2007. How does modifying a DEM to reflect known hydrology affect subsequent terrain analysis? Journal of Hydrology 332 :30-39.

Centre d'Études de Géographie Tropicale (CEGET), 1985. Atlas d'Haïti, 146 pp.

Charleux-Demargne J., 2001. Qualité des Modèles Numériques de Terrain pour l'Hydrologie. Application à la Caractérisation du Régime de Crues des Bassins Versants. PhD thesis, University of Montpellier, France, 350 pp.

De Jager A.L., Vogt J.V., 2010. Development and demonstration of a structured hydrological feature coding system for Europe. Hydrological Sciences Journal 55(5) :661-675.

El Hage M., 2012. Etude de la qualité géomorphologique de modèles numériques de terrain issus de l'imagerie spatiale. PhD thesis, Conservatoire National des Arts et Métiers, Paris, France, $146 \mathrm{pp}$.

Frelat R., Claden M., Bendraoua F., El Hage M., Polidori L., Gaucherel C., 2012. Projet BVH Phase I : Description spatiale des bassins versants d'Haïti. Technical Report, Institut Français de Pondichéry, India, 85 pp.
Gyasi-Agyei Y., Willgoose G., de Troch F.P., 1995. Effects of vertical resolution and map scale of digital elevation models on geomorphological parameters used in hydrology. Hydrological Processes 9 :363-382.

Hosseinzadeh S.R., 2011. Assessing the quality of ASTER DEMs for Hydrological Applications (Case Study : Cheshmehkhan Catchment in the northeastern of Iran). In : International Conference on Environment Science and Engineering, IPCBEE, Bali Island, Indonesia, 1-3 April 2011, vol.8, pp. 37-41.

Labbé S., Linarès S., Pausader M. Rolland K., 2012. Réalisation du référentiel hydrographique BD Carthage, sur le bassin de la Guyane à partir de données de télédétection. In : XV SELPER Symposium, Cayenne, France, 19-23 November 2012.

McMaster K.J., 2002. Effects of digital elevation model resolution on derived stream network positions. Water Resources Research 38(13) :1-8.

O'Callaghan J F., Mark D.M., 1984. The extraction of drainage networks from digital elevation data. Computer Vision, Graphics, and Image Processing 28(3) :323-344. Pella H., Lejot J., Lamouroux N., Snelder T., 2012. Le réseau hydrographique théorique $(\mathrm{RHT})$ français et ses attributs environnementaux. Géomorphologie : relief, processus, environnement, $n^{\circ} 3$, pp. 317-336.

Polidori L., 1995. Réflexions sur la qualité des modèles numériques de terrain. Bulletin de la SFPT 139 :10-19.

Polidori L., Chorowicz J., Guillande R., 1991. Description of terrain as a fractal surface and application to digital elevation model quality assessment. Photogrammetric Engineering \& Remote Sensing 57(10):1329-1332.

Potere D., 2008. Horizontal Positional Accuracy of Google Earth's High-Resolution Imagery Archive. Sensors 8(12) :7973-7981.

Rodriguez-Iturbe I, Rinaldo A., 1997. Fractal River Basins : Chance and Self-Organization. Cambridge University Press : New York, USA.

Saunders W., 1999. Preparation of DEMs for use in environmental modeling analysis. Esri User Conference, San Diego, California, USA, pp.1-18.

Tarboton D.G., Rodriguez-Iturbe I., 1991. On the extraction of channel networks from digital elevation data. Hydrological Processes 5(1) : 81-100.

Verdin K.L., Verdin J.P., 1999. A topological system for delineation and codification of the Earth's river basins. Journal of Hydrology $218: 1-12$.

Yousefzadeh M., Mojaradi B., 2012. Combined rigorousgeneric direct orthorectification procedure for IRS-p6 sensors. ISPRS Journal of Photogrammetry and Remote Sensing $74: 122-132$. 


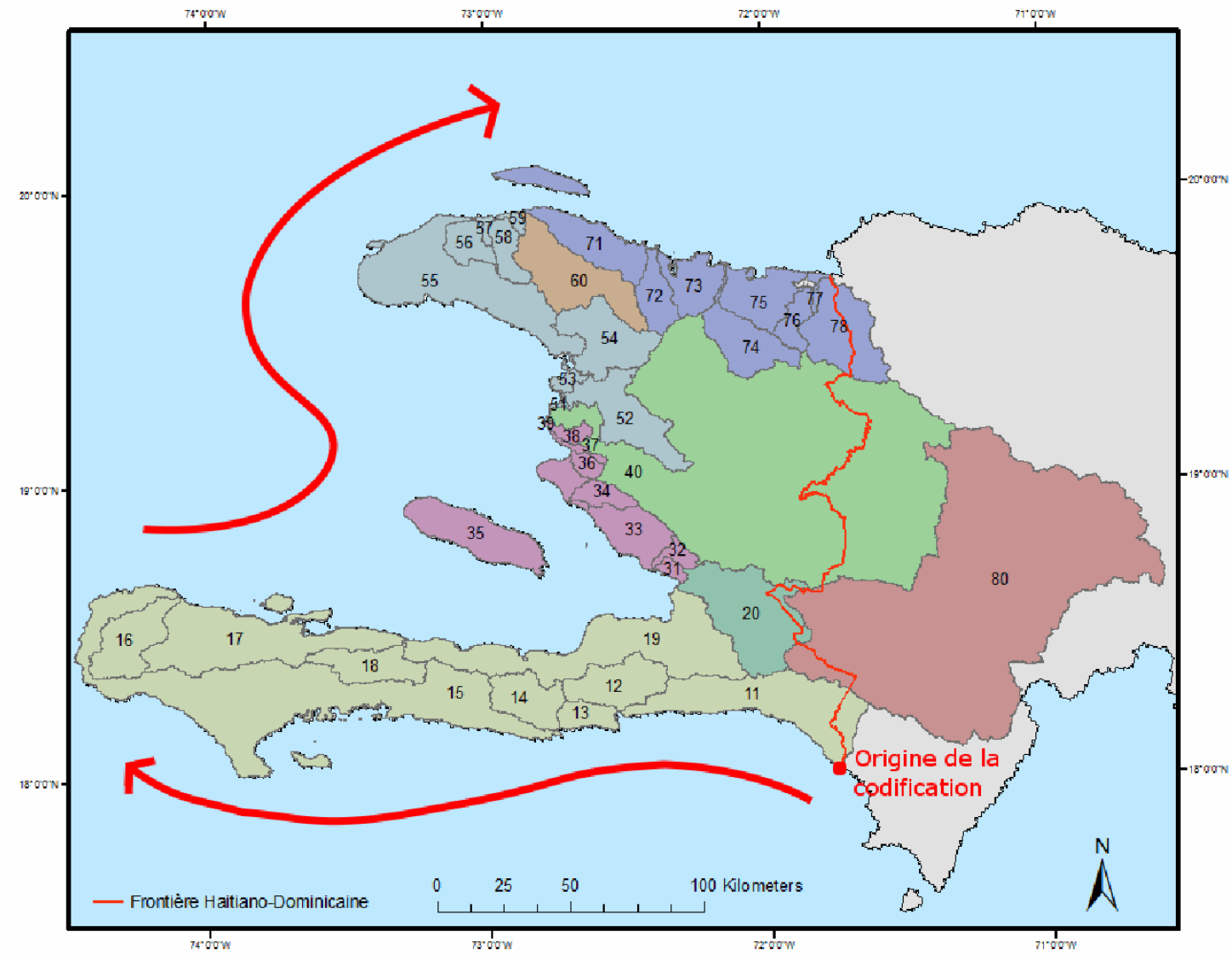

FIGURE 9 : Mise en place de la codification de Pfafstetter sur notre zone d'étude, pour les 20 plus grands bassins versants ou jusqu'à la subdivision aux deux premiers chiffres du code. Les couleurs montrent l'ordre 1 de la codification de Pfafstetter et les chiffres présentent la subdivision à l'ordre 2. 